\title{
Cartographie de la dynamique de terroirs villageois à l'aide d'un drone dans les aires protégées de la République démocratique du Congo
}

\author{
Jean Semekı NgabinzeKE ${ }^{1,2}$ \\ Julie LINCHANT ${ }^{3}$ \\ Samuel QUEVAUVILLERS ${ }^{4}$ \\ Jean-Marie Kahindo MuHONGYA \\ Philippe LeJEUNE ${ }^{4}$ \\ Cédric Vermeulen ${ }^{3}$
}

${ }^{1}$ Université de Kisangani

Faculté de gestion des ressources naturelles

renouvelables

Département des eaux et forêts

BP 2012, Kisangani

République démocratique du Congo

${ }^{2}$ Université de Kinshasa

Faculté des sciences agronomiques

Département de gestion des ressources

naturelles

BP 117, Kinshasa XI

République démocratique du Congo

${ }^{3}$ Université de Liège Gembloux

Agro-Bio Tech

Unité de recherche TERRA

Passage des Déportés, 2

B-5030 Gembloux

Belgique

${ }^{4}$ Université de Liège Gembloux

Agro-Bio Tech

Unité de recherche BIOSE

Passage des Déportés, 2

B-5030 Gembloux

Belgique

${ }^{5}$ Université de Kisangani

Faculté des sciences

Département de botanique

BP 2012, Kisangani

République démocratique du Congo

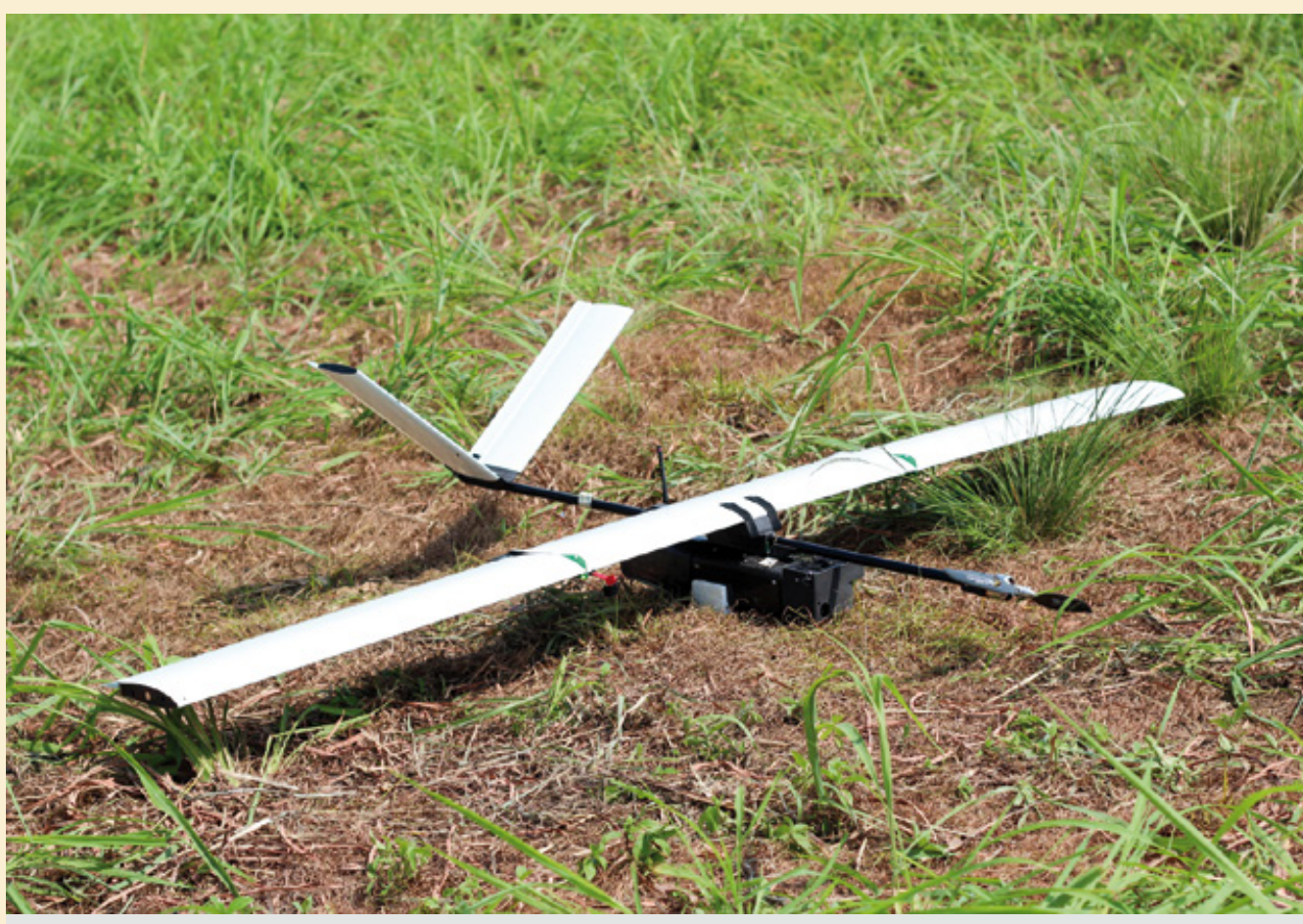

Photo 1.

Le drone Falcon utilisé pour la cartographie de l'occupation des sols à Gbere.

Photo J. Linchant. 


\section{RÉSUMÉ}

\section{CARTOGRAPHIE DE LA DYNAMIQUE DE TERROIRS VILLAGEOIS À L'AIDE D'UN DRONE DANS LES AIRES PROTÉGÉES DE LA RÉPUBLIQUE DÉMOCRATIQUE DU CONGO}

Les aires protégées de la République démocratique du Congo (RDC) sont menacées par diverses pressions anthropiques nécessitant un suivi fréquent et précis. Le mini-drone Falcon équipé d'un appareil photo numérique Sony NEX-7 a été utilisé pour cartographier et suivre la dynamique d'un terroir villageois dans le Domaine de chasse de Mondo Missa à l'est du Parc national de la Garamba, au nord-est de la RDC. Un total de 3143 photos acquises en avril et juillet 2015, avec une résolution au sol de $8 \mathrm{~cm} /$ pixel, a été orthorectifié. La cartographie a porté sur une zone de 114 ha. Les ortho-images ont d'abord été segmentées, les segments étant ensuite classés manuellement par photo-interprétation. Des changements notables ont été constatés entre les deux dates. Les zones des forêts et savanes ont perdu 6,5 ha (86,6 à 80,1 ha). Les jachères sont passées de 16,9 à 8,2 ha, les défriches de 4,1 à 10,0 ha. Les cultures saisonnières ont connu une variation allant de 3,2 à 11,8 ha. La taille moyenne des parcelles cultivées est de 0,2 ha $(\sigma=0,14$ ha ; $n=50)$. Enfin, la surface occupée par les arbres isolés a peu évolué (de 1,3 à 1,9 ha), celle des implantations humaines étant constante $(1,7 \mathrm{ha})$. Ces résultats traduisent le fait que l'expansion de l'agriculture itinérante sur brûlis induit une conversion des habitats naturels et une modification de la composition végétale. Les aéronefs sans pilote à bord permettent de réaliser une cartographie précise et une surveillance rapide des changements d'affectation des terres à petite échelle dans les aires protégées des forêts et savanes tropicales. Ils offrent donc une solution efficace pour évaluer la déforestation et la dégradation au sein des espaces occupés par les communautés locales. Cette évaluation représente un enjeu important dans le processus REDD+ qui envisage de quantifier avec précision ces évolutions.

Mots-clés : terroir villageois, utilisation des terres, agriculture itinérante sur brûlis, détection des changements, conversion de l'habitat, fragmentation, drone, aires protégées, République démocratique du Congo.

\section{ABSTRACT}

\section{USING DRONE TECHNOLOGY TO MAP VILLAGE LANDS IN PROTECTED AREAS OF THE DEMOCRATIC REPUBLIC OF CONGO}

Protected areas of the Democratic Republic of Congo (DRC) are under threat from various human pressures that need to be monitored accurately and on a regular basis. A small Falcon drone mounted with a Sony NEX-7 camera was used for mapping missions to track the dynamics of village lands in the Mondo Missa Game Reserve, which lies along the eastern border of Garamba National Park in north-eastern DRC. The mapping missions collected a total of 3,143 photos taken in April and July 2015, covering an area of 114 ha with a ground resolution of $8 \mathrm{~cm} /$ pixel. The images were then ortho-rectified, and the resulting ortho-images segmented. The segments were then classified manually by photo-interpretation. Significant changes had occurred between the two periods: 6.5 ha of natural forest and savannah had been lost (declining from 86.6 to $80.1 \mathrm{ha}$ ), fallow areas had increased from 16.9 to 8.2 ha and cleared land from 4.1 to 10.0 ha. The variations in seasonal crop areas ranged from 3.2 to $11.8 \mathrm{ha}$. The average size of cultivated plots was 0.2 ha ( $\sigma=0.14$ ha, $\mathrm{n}=50$ ). Finally, the area covered by isolated trees had increased very slightly (from 1.3 to 1.9 ha) while the area occupied by human settlements remained constant (1.7 ha). These results show that the expansion of itinerant slash-and-burn cultivation results in the conversion of natural habitats and modifies plant communities. Unmanned aerial systems can thus help to produce accurate maps and undertake rapid monitoring of small-scale land use changes in protected areas in tropical forests and savannah. They offer an efficient solution for assessments of deforestation and degradation in areas used by local communities. Such assessments are an issue of considerable importance in the REDD+ process, which is aiming to quantify these changes accurately.

Keywords: village lands, land use, itinerant slash-and-burn cultivation, detection of changes, habitat conversion, fragmentation, drone, protected areas, Democratic Republic of Congo.

\section{CARTOGRAFíA DE LA DINÁMICA DE TIERRAS COMUNALES MEDIANTE UN DRON EN LAS ÁREAS PROTEGIDAS DE LA REPÚBLICA DEMOCRÁTICA DEL CONGO}

Las áreas protegidas de la República Democrática del Congo (RDC) sufren la amenaza de diversas presiones antrópicas que requieren un seguimiento regular y detallado. Se utilizó el minidrón Falcon, equipado con una cámara fotográfica digital Sony nex7, para cartografiar y seguir la dinámica de una tierra comunal en la reserva de caza de Mondo Missa en la parte oriental del Parque Nacional Garamba, en el noreste de la RDC. Se tomaron un total de 3143 fotos en abril y julio de 2015 con una resolución topográfica de $8 \mathrm{~cm} /$ pixel y se ortorrectificaron. La cartografía cubre una zona de 114 ha. Primero se segmentaron las ortoimágenes y después se clasificaron los segmentos manualmente por fotointerpretación. Se observaron cambios significativos entre los dos períodos. Las zonas de bosques y sabanas perdieron 6,5 ha (86,6 a 80,1 ha). Los barbechos pasaron de 16,9 a 8,2 ha y las tierras desbrozadas de 4,1 a 10,0 ha. Los cultivos estacionales variaron de 3,2 a 11,8 ha. El tamaño promedio de las parcelas cultivadas es de 0,2 ha $(\sigma=0,14$ ha, $\mathrm{n}=50)$. Por último, el área cubierta por árboles aislados evolucionó poco (de 1,3 a 1, 9 ha), mientras que la de los asentamientos humanos se mantuvo constante (1,7 ha). Estos resultados revelan que la expansión de la agricultura itinerante de roza y quema provoca una conversión de los hábitats naturales y una modificación de la composición vegetal. Las aeronaves no tripuladas permiten realizar una cartografía detallada y un control rápido de los cambios de uso del suelo a pequeña escala en las áreas protegidas de boques y sabanas tropicales. Proporcionan, por tanto, una solución eficaz para evaluar la deforestación y degradación en los espacios ocupados por las comunidades locales. Esta evaluación supone un importante aspecto en el proceso REDD+ que aspira a cuantificar con precisión estos cambios.

Palabras clave: tierras comunales, uso del suelo, agricultura itinerante de roza y quema, detección de cambios, conversión del hábitat, fragmentación, dron, áreas protegidas, República Democrática del Congo. 


\section{Introduction}

La République démocratique du Congo (RDC) abrite un des réservoirs de biodiversité les plus importants au monde (Pelissier et al., 2015). Les aires protégées qui couvrent près de $11 \%$ du territoire national constituent la pierre angulaire de la conservation de cette diversité biologique (Eba'a Atyi et Bayol, 2009 ; ICCN, 2012).

Toutefois, les conflits armés récurrents que connaît le pays depuis plusieurs décennies, associés à la paupérisation des populations locales, ont intensifié les menaces sur ces aires protégées (Unesco, 2010 ; Misser, 2013). Celles-ci sont aujourd'hui parmi les plus vulnérables au monde (Butsic et al., 2015). En RDC, la perte de couvert forestier au sein des aires protégées a en effet augmenté de $64 \%$ entre 2000 et 2010 (Potapov et al., 2012). La principale cause de ce processus est l'agriculture itinérante sur brûlis qui constitue la première forme d'agriculture du pays (Ickowitz et al., 2015). Cette pratique donne lieu à la création de petites clairières isolées dans les forêts intactes, conduisant à la fragmentation de l'habitat (Mayaux et al., 1999 ; Nackoney et al., 2014 ; Molinario et al., 2015). Dans ce contexte, la surveillance des aires protégées par l'Institut congolais pour la conservation de la nature (ICCN) constitue un véritable défi, en raison de l'importance des surfaces concernées et de la faiblesse des moyens disponibles (UICN/PACO, 2010 ; ICCN, 2012 ; Misser, 2013).

L'essentiel des travaux réalisés pour quantifier la perte du couvert forestier en RDC s'appuie sur l'imagerie satellitaire, principalement les images Landsat (Potapov et al., 2012). La télédétection est en effet un outil efficace pour surveiller l'évolution de l'occupation des sols et détecter les changements d'habitat notamment au sein des aires protégées (Spanhove et al., 2012 ; Nagendra et al., 2015), que ce soit en relation avec l'évolution démographique et l'urbanisation (Svancara et al., 2009 ; Wang et al., 2009), l'expansion des cultures (Phalan et al., 2013), l'impact des conflits armés, ou encore l'exploitation minière (Butsic et al., 2015).

Cette technologie présente cependant plusieurs limites. Parmi celles-ci, on peut citer les coûts d'acquisition dans le cas des satellites commerciaux à très haute résolution (GeoEye, Ikonos, Pléiades, Worldview) (Wellens et al., 2013 ; Willis, 2015), mais aussi la résolution spatiale et la résolution temporelle limitées dans le cas des images à haute résolution (Landsat) accessibles gratuitement (Wiens et al., 2009 ; Kakaes et al., 2015). Ces deux derniers problèmes sont particulièrement contraignants dans le cas de la surveillance d'empiètements au sein d'aires protégées qui opèrent à petite échelle (Nagendra et al., 2013 ; Kakaes et al., 2015).
Il existe donc un besoin réel pour des outils adaptés à la surveillance des perturbations anthropiques au sein d'aires protégées à l'échelle locale (entendue ici comme la taille de la zone considérée ou des objets au sol ; Wulder et al., 2004), notamment celles liées à l'agriculture itinérante sur brûlis. Malgré leurs faibles étendues allant de 0,25 ha dans la forêt d'Ituri (Wilkie et Finn, 1990) à 1,4 ha au niveau national (Potapov et al., 2012), ces perturbations constituent une réelle menace pour la conservation de la biodiversité. Les outils de surveillance doivent permettre un suivi précis et continu des installations humaines au sein d'aires protégées pour que leurs gestionnaires soient en mesure de prendre rapidement des décisions adaptées. Les informations ainsi récoltées peuvent également servir de base de discussion dans les approches participatives.

Alors qu'elle trouve ses origines dans le domaine militaire, la technologie des aéronefs télécommandés sans pilote à bord (unmanned aerial system), plus communément appelés drones (de l'anglais drone signifiant faux bourdon), est de plus en plus utilisée dans le domaine de la gestion des ressources naturelles (Shahbazi et al., 2014), y compris la gestion et la surveillance des pâturages (Rango et al., 2009 ; Laliberte et al., 2010), l'évaluation de la biodiversité en forêt (Getzin et al., 2012), la surveillance des incendies (Merino et al., 2012) et de la structure des écosystèmes (Puttock et al., 2015 ; Faye et al., 2015), l'agriculture de précision (Torres-Sánchez et al., 2015), et les comptages de faune (Linchant et al., 2015). L'utilisation des drones pour la détection d'activités illégales a déjà été abordée (Koh et Wich, 2012 ; Paneque-Galvez et al., 2014 ; Semeki et al., 2016). Cependant, les applications de cette technologie pour la conservation, notamment la détection des changements d'affectation des terres, sont encore à explorer (Kakaes et al., 2015), particulièrement en zones tropicales.

L'objectif de cette étude est de concevoir et de tester une approche simple et rapide permettant de cartographier et de surveiller les terroirs villageois au sein des aires protégées de la RDC à l'aide d'un mini-drone. Les objectifs spécifiques de ce travail consistent (1) à évaluer l'efficacité des opérations de collecte et de traitement des images acquises par le drone, (2) à proposer une méthode de cartographie fine et rapide de l'utilisation des terres et de sa dynamique temporelle et (3) à analyser les résultats obtenus sur une zone test. 


\section{Matériel et méthodes}

\section{Site d'étude}

L'étude s'est déroulée dans le village de Gbere, au sein du Domaine de chasse de Mondo-Missa situé à l'est du Parc national de la Garamba (PNG), dans le nord-est de la République démocratique du Congo, à la frontière avec le Soudan du Sud (figure 1). La superficie du domaine est d'environ $1983 \mathrm{~km}^{2}$ (Misser, 2013). Le climat est du type tropical semi-humide (soudano-guinéen) (AW3) de la classification de Koppen (De Saeger, 1954). Ce climat présente beaucoup de variabilité. Il est caractérisé par une grande saison sèche très aride de décembre à février ; les précipitations s'étendent sur neuf mois (mars à novembre) et marquent une diminution de juin à juillet. Le mois de novembre peut aussi être sec. La pluviométrie est de $1500 \mathrm{~mm}$ en moyenne par an et la température moyenne annuelle est de $25^{\circ} \mathrm{C}$ (Balimbaki, 2015).

Les formations végétales comprennent les savanes herbeuses et arborées marquées par la présence de Loudetia arundinacea, Hyparrhenia sp., Kigelia africana, Terminalia mollis, Terminalia avicennioides, avec une prédominance des forêts claires, forêts denses sèches et des galeries forestières composées essentiellement de Irvingia smithii, Erythrophleum suaveolens, Milicia excelsa, Khaya grandifoliola et Ficus trichopoda (De Saeger, 1954). Les principales espèces de grands mammifères d'intérêt touristique sont l'éléphant (Loxodonta africana, Loxodonta cyclotis), la panthère (Panthera leo), le bongo (Tragelephus euryceros) et la girafe (Giraffa camelopardalis antiquorum) (Balimbaki, 2015).

Le Parc national de la Garamba est inscrit au Patrimoine de l'Humanité par l'Unesco depuis 1981. En 1995, il a été inclus dans la liste des sites en péril en raison de l'intensification du braconnage pratiqué par les populations locales et les groupes armés (Unesco, 2010 ; Misser, 2013). En outre, diverses activités non réglementées (agriculture sur brûlis, orpaillage, carbonisation) sont en expansion dans les domaines de chasse qui constituent des zones tampons du parc (Kayungura, 2009; Balimbaki, 2015). C'est notamment le cas pour le village de Gbere où l'agriculture itinérante sur brûlis pour la production de cultures vivrières constitue la principale activité pour la survie des populations locales.

Le système cultural pratiqué dans cette région s'apparente à l'agriculture itinérante sur brûlis (figure 2). Les défrichements partiels (certains arbres sont conservés) de la forêt primaire sont réalisés en saison sèche. Les espaces déboisés sont ensuite brûlés avant de procéder à la préparation des champs et au semis qui se déroulent en mars-avril pour la première récolte et en août-septembre pour la seconde récolte. Le riz (Oryza sativa), le maïs (Zea mays), le haricot (Phaseolus vulgaris), l'arachide (Arachis hypogaea) (deux récoltes par an) et le manioc (Manihot esculenta) (récolté progressivement après 10 à 12 mois) constituent les principales cultures (Balimbaki, 2015). Une première et courte période de friche

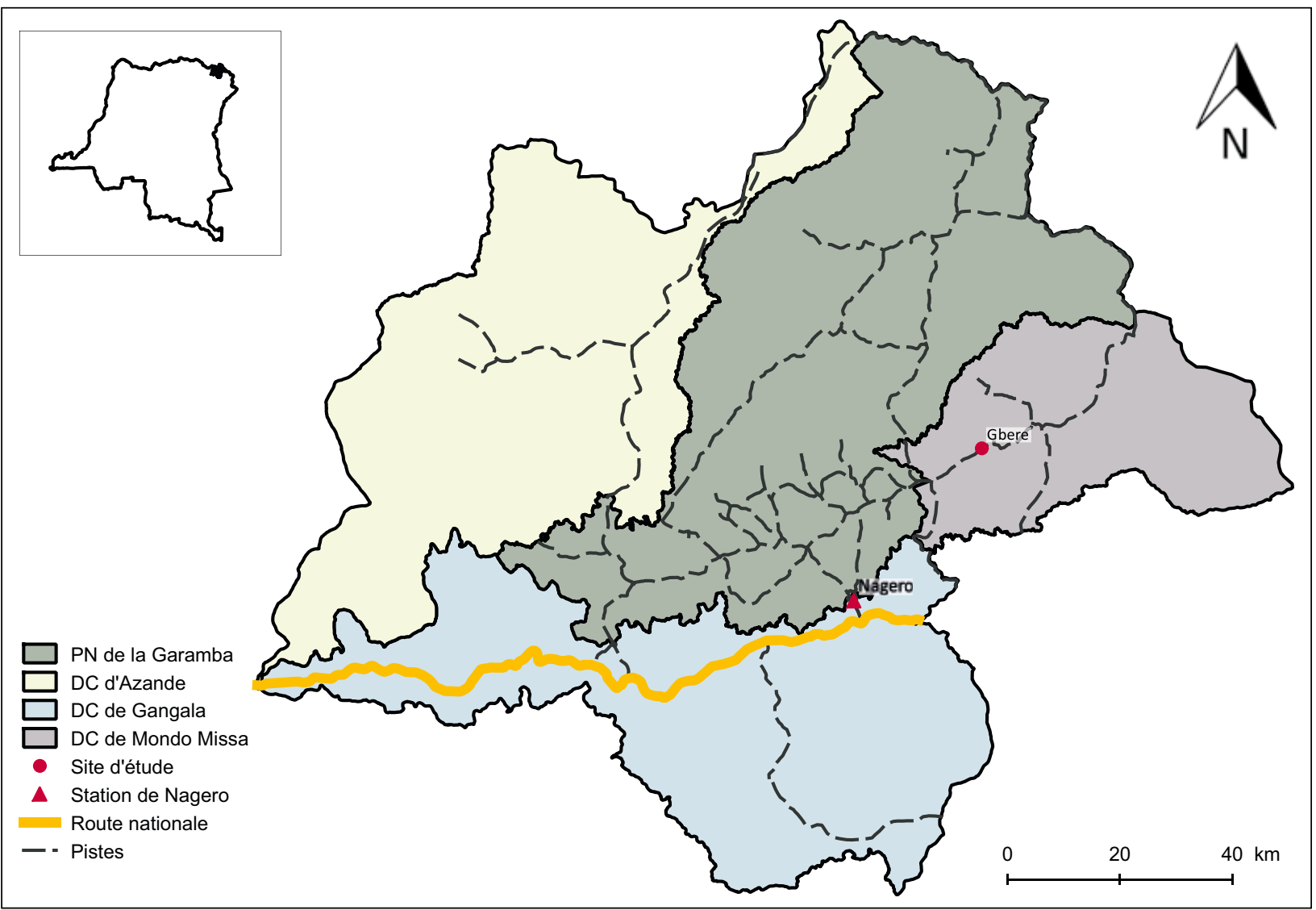

Figure 1.

Localisation de la zone d'étude dans le complexe de la Garamba, situé dans le nord-est de la République démocratique du Congo. 


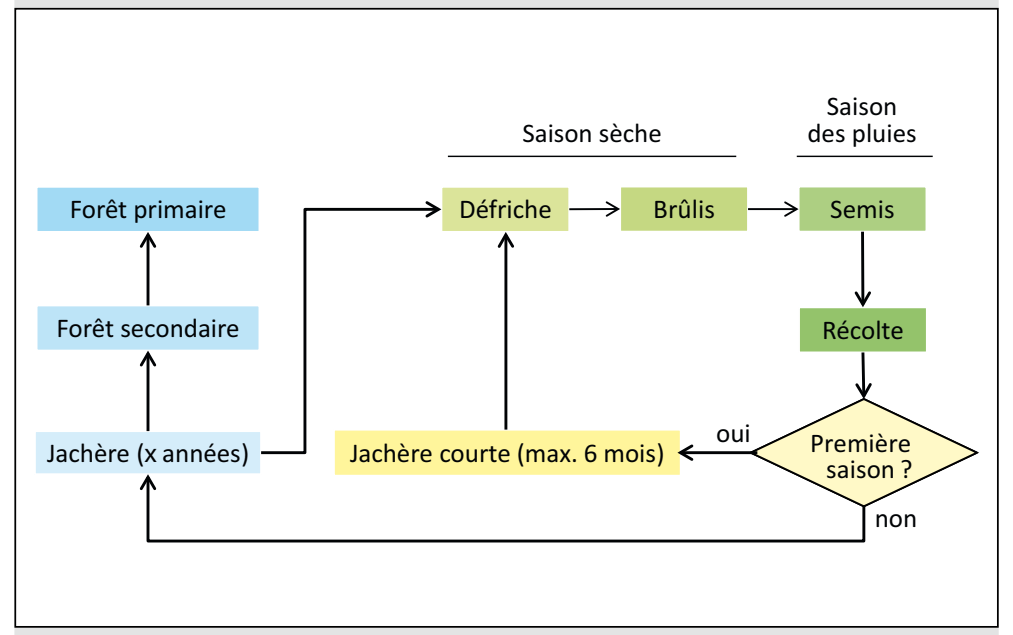

Figure 2.

Représentation schématique du système cultural en place dans le village de Gbere.

\section{Acquisition des images}

Les vols ont été réalisés respectivement le $1^{\text {er }}$ avril 2015 et le 21 juillet 2015 sur le terroir du village de Gbere. La première date correspond à la fin de la campagne agricole dont la récolte a été effectuée en décembre 2014 et le début des travaux champêtres de la saison suivante. La seconde date correspond à la période de maturité pour la plupart des cultures saisonnières. La zone d'étude a été délimitée à l'issue d'une cartographie participative menée avec les villageois selon la méthode proposée par Larzillière et al. (2013).

Pour les deux missions, les images ont été acquises à une hauteur de vol de $250 \mathrm{~m}$ induisant une résolution au sol de $8 \mathrm{~cm} / \mathrm{pixel}$ et une emprise au sol de 297 m x 206 m par image. Les images ont été acquises dans le spectre lumineux du visible rouge, vert et bleu (RVB). Une cadence d'une prise de vue tous les $20 \mathrm{~m}$ a permis d'obtenir un recouvrement longitudinal de $90 \%$. L'espacement entre les lignes de vol permet quant à lui un recouvrement latéral de $70 \%$. Ces recouvrements importants garantissent un bon assemblage des images lors du traitement photogrammétrique (Semeki et al., 2016). La vitesse d'obturation de la caméra a été réglée sur $1 / 1600$ et $1 / 2000$, et la sensibilité Iso fixée à 400 et 200 respectivement pour le premier et second vol en fonction des conditions de luminosité au moment de la mission. Les deux plans de vol, constitués de onze transects parallèles, ont permis de survoler une surface de $5,5 \mathrm{~km}^{2}$.

Une série de 329 points GPS a été levée sur l'emprise des zones anthropisées (champs, jachères) aux mêmes dates que les deux vols, à l'aide d'un récepteur Garmin GPSmap 62s. La précision de ce dernier, dans la zone d'étude (terrain découvert), peut être évaluée à environ $3 \mathrm{~m}$. Les points relevés ont servi à l'interprétation visuelle des images.

\section{Traitement des images}

La procédure de traitement des images comprend les étapes de géoréférencement, d'orthorectification, de mosaïquage, de découpage et de segmentation (figure 3). Chacune de ces étapes est décrite dans les lignes qui suivent.

Le géoréférencement des images a été effectué à partir des informations de vol fournies par le logiciel de planification Mission Planner (version 1.3.31), après avoir synchronisé l'horloge de la caméra avec celle du GPS du drone.

Le logiciel Agisoft PhotoScan Professionnal (version 1.1.2) a ensuite été utilisé pour l'orthorectification et la création des mosaïques. Le processus comporte trois étapes qui sont respectivement (1) l'alignement des images (alignement), (2) la construction du modèle 3D (dense cloud) permettant ensuite (3) de générer un modèle numérique de surface (MNS) et une ortho-image (Torres-Sánchez et al., 2015). L'ensemble du processus est entièrement automatisé après avoir chargé les images et le fichier de localisation de celles-ci.

${ }^{1}$ www.falconunmanned.com 


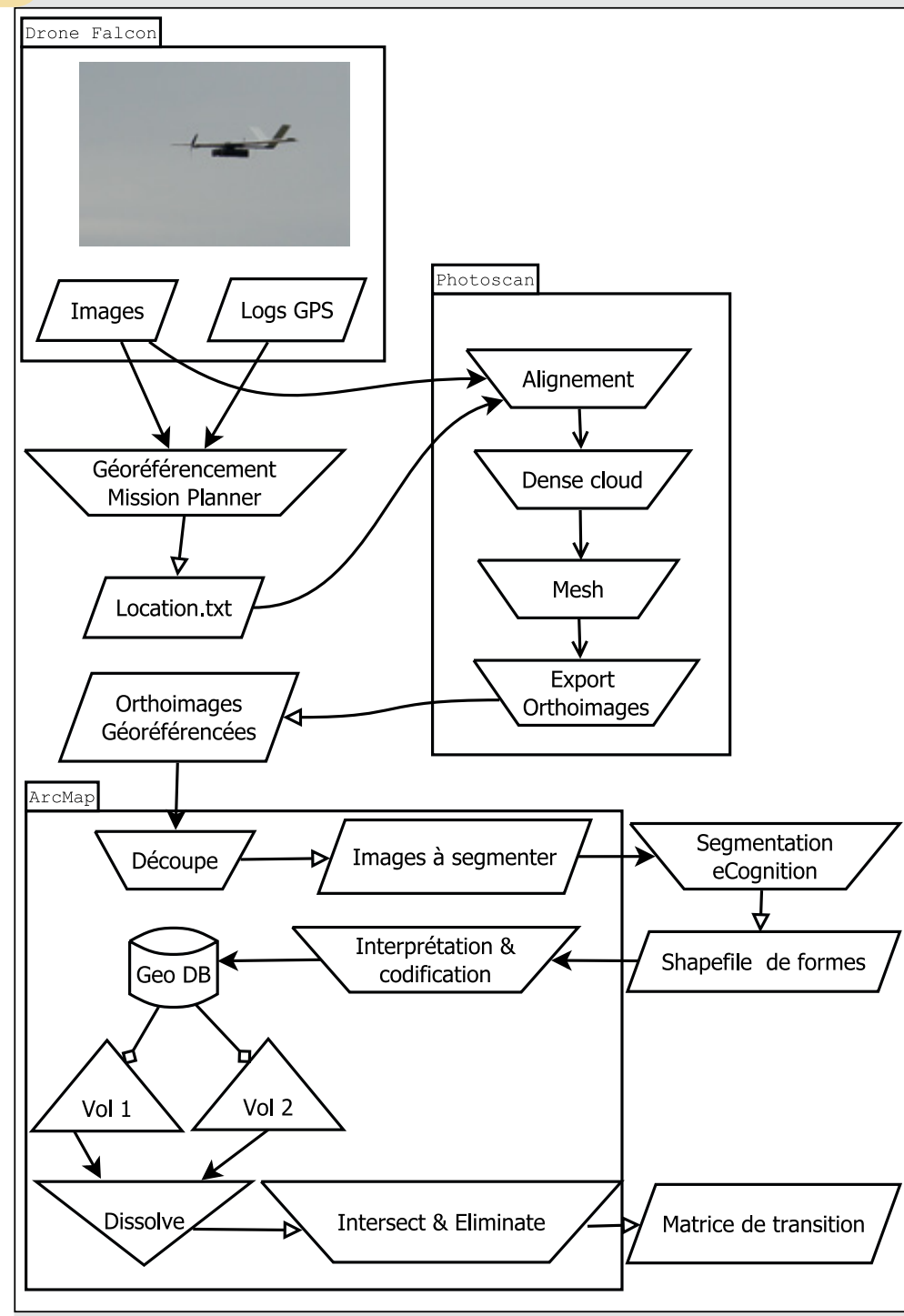

Figure 3.

Procédure de traitement et d'analyse d'images réalisée pour étudier la dynamique d'occupation des sols.

Un masque de forme rectangulaire a été créé pour découper les deux mosaïques et définir précisément l'emprise de la zone d'intérêt centrée sur les zones anthropisées du village de Gbere. Cette zone d'étude couvre une superficie de 114 ha.

Les deux ortho-images ainsi découpées ont été segmentées en utilisant l'algorithme de segmentation multirésolution du logiciel eCognition Developer $9^{2}$. Un facteur d'échelle de 45 et des facteurs de forme et de compacité fixés tous les deux à 0,5 ont été retenus après différents essais, l'objectif étant d'éviter autant que possible le phénomène de sous-segmentation, sans pour autant augmenter de manière trop importante le nombre de segments. Cette phase de segmentation a abouti à la production d'une couche de polygones pour chaque date. Ces couches ont été stockées dans une géodatabase personnelle ESRI pour les traitements et analyses ultérieurs.

\footnotetext{
${ }^{2}$ www.ecognition.com
}

\section{Analyse des images}

Les couches de segments ainsi produites pour les deux dates ont été interprétées visuellement par un même opérateur ayant une bonne connaissance du terrain. L'approche cartographique basée sur une interprétation visuelle et non sur un processus de classification a été privilégiée dans cette étude. Ce choix se justifie par la très haute résolution spatiale des images $(10 \mathrm{~cm} /$ pixel), la faible résolution spectrale (image RVB seulement), mais aussi la très grande hétérogénéité des éléments à cartographier et la volonté d'être capable de discerner les différents types de culture. Dans ces conditions, on peut admettre l'intérêt de recourir à une démarche de photo-interprétation réalisée par un opérateur ayant une bonne connaissance du terrain. La phase de segmentation des ortho-images a permis de faciliter et d'objectiver le travail de délimitation des unités d'occupation/utilisation des sols.

La légende considérée pour cet exercice est structurée en deux niveaux (tableau I).

La classe " arbres isolés » correspond à des reliques de forêts (bosquets) au sein de zones anthropisées. Le choix de globaliser les formations forestières et les savanes se justifie par le fait que l'étude se focalise sur le monitoring des activités anthropiques et tente de proposer une méthode de cartographie rapide et simple à mettre en œuvre. Les points GPS relevés sur le terrain ont été particulièrement utiles pour l'apprentissage de la reconnaissance des cultures saisonnières préalablement à la phase d'interprétation. Lors de la phase d'interprétation des images et de codage des polygones, l'opérateur avait la possibilité de redécouper manuellement les polygones présentant un problème de sous-segmentation. Une trace de ces modifications a été conservée dans la table d'attributs de la couche vectorielle.

Àl'issue de la phase d'interprétation et de codification des polygones, chaque couche a fait l'objet d'une fusion des polygones correspondant à la même occupation des sols (commande dissolve). Les deux couches vectorielles ont ensuite été croisées (commande intersect) pour mettre en évidence les changements d'occupation des sols. Afin d'alléger quelque peu la couche résultante et de faciliter les analyses ultérieures, les polygones de taille inférieure à $5 \mathrm{~m}^{2}$ (sliver polygons) ont été rattachés au polygone voisin le plus important à l'aide de la commande eliminate.

La matrice de transition, qui a pour finalité de synthétiser la dynamique d'occupation des sols entre les deux dates, a été construite à l'aide de requêtes SQL au sein de la géodatabase contenant les deux couches cartographiques.

Tous les géotraitements ont été réalisés avec la version 10.2 du logiciel ArcGIS . Les requêtes SQL ont été réalisées avec le logiciel Access 2010.

Par ailleurs, une estimation de la taille moyenne des parcelles cultivées a été effectuée par digitalisation à l'écran des limites de parcelles observées sur l'image de juillet. L'approche a consisté à digitaliser les limites de 50 parcelles sélectionnées à l'aide d'une série de points générés aléatoirement au sein des parcelles cultivées. 


\begin{tabular}{|c|c|c|}
\hline \multicolumn{3}{|c|}{$\begin{array}{l}\text { Tableau I. } \\
\text { Légende utilisée pour l'interprétation des ortho-images et la création des cartes d'occupation des sols. }\end{array}$} \\
\hline $\mathbf{N}^{\circ}$ & Classe & Sous-classe \\
\hline 1 & Arbres isolés & - \\
\hline \multirow[t]{2}{*}{2} & Cultures pérennes & Bananier \\
\hline & & Manguier \\
\hline \multirow[t]{13}{*}{3} & Cultures saisonnières & Arachide-maïs \\
\hline & & Arachide \\
\hline & & Haricot \\
\hline & & Haricot-riz \\
\hline & & Maïs \\
\hline & & Manioc \\
\hline & & Manioc-arachide \\
\hline & & Manioc-maïs \\
\hline & & Patate douce \\
\hline & & Patate douce-manioc-maïs \\
\hline & & Riz \\
\hline & & Riz-maïs \\
\hline & & Semis \\
\hline \multirow[t]{4}{*}{4} & Défriches & Zone récemment déboisée \\
\hline & & Zone récemment désherbée \\
\hline & & Zone récemment brûlée \\
\hline & & Zone récemment labourée \\
\hline 5 & Forêts et savanes & - \\
\hline \multirow[t]{3}{*}{6} & Implantations humaines & Habitations \\
\hline & & Sol nu \\
\hline & & Sol peu végétalisé (abord des maisons et des pistes) \\
\hline \multirow[t]{4}{*}{7} & Jachères & Jeunes jachères (jachères courtes ou champs récoltés lors de la précédente campagne) \\
\hline & & Formations herbeuses avec présence de Chromolaena odorata \\
\hline & & Formations herbeuses avec présence d’un tapis graminéen \\
\hline & & Vieilles jachères (champ récolté il y a plus d’un an) \\
\hline
\end{tabular}

\section{Résultats}

\section{Temps de mise en œuvre}

L'acquisition des données (deux vols : 3143 photos, 1431 pour le vol d'avril et 1712 pour celui de juillet) a nécessité l'intervention de deux personnes pendant trois heures, soit un total de six heures. Le temps requis pour la prise de points GPS sur les champs est de six heures par opérateur pour les deux dates.

Le traitement des images acquises lors des deux vols a été effectué sur un poste de travail muni d'une mémoire Ram de 65 Go. La durée totale de ces traitements est d'environ sept heures, réparties en $4 \mathrm{~h} 30$ min pour l'orthorectification et la création des mosaïques, et $2 \mathrm{~h} 30$ min pour la segmentation. On peut considérer que l'intervention de l'opérateur dans cette étape ne représente que deux heures de travail.
La phase d'interprétation a nécessité quant à elle un total de 45 heures de travail. II s'agit du poste le plus important, puisqu'il représente $76 \%$ du temps de travail « opérateur ». Cette proportion s'explique à la fois par le degré de détail élevé de la légende utilisée et par le fait que l'opérateur, bien qu'ayant une très bonne connaissance du terrain, a dû passer par une phase d'apprentissage du processus d'interprétation et de codage des polygones en début d'étude.

\section{Traitement photogrammétrique et segmentation des images}

Les deux lots d'images ont été correctement géoréférencés à partir des données de position fournies par le logiciel Mission Planner. Les erreurs de reprojection associées au processus d'assemblage des images par Photoscan sont respectivement de 1,34 et 1,59 pixels pour les vols d'avril et de juillet. Les proportions d'images correctement alignées 


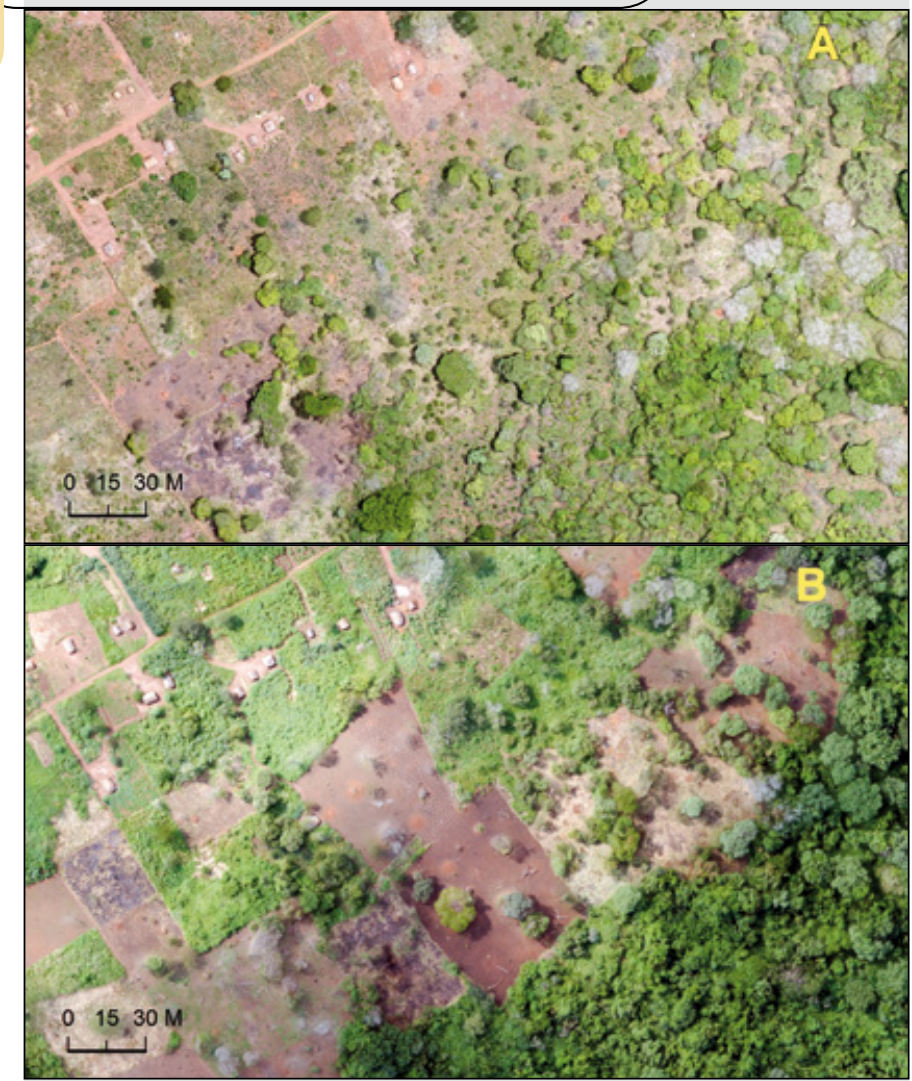

Figure 4.

Extrait des ortho-images illustrant un paysage fortement anthropisé dans le village de Gbere : (a) en avril 2015 et (b) en juillet 2015. par le logiciel Photoscan sont de $99,4 \%$ et $96,7 \%$, respectivement pour les vols d'avril et juillet. Les images rejetées étaient généralement floues ou trop sombres. Les orthoimages ont été exportées de Photoscan avec une résolution de $10 \mathrm{~cm} /$ pixel. La figure 4 illustre une portion de ces orthoimages présentant un faciès fortement anthropisé.

L'étape de segmentation des images sur la zone d'intérêt a produit 172829 et 70952 polygones, respectivement pour le vol d'avril et le vol de juillet (figure 5). Pour le premier vol, 216 polygones ont été redécoupés manuellement, soit $0,12 \%$ du nombre total, et 598 polygones pour le second vol, soit $0,84 \%$ de l'ensemble.

\section{Cartographie du terroir villageois et de sa dynamique}

Le résultat principal de cette recherche est la production d'une cartographie très détaillée et précise du terroir villageois étudié et de son évolution entre les deux dates considérées (figure 6). La matrice de transition construite sur la base de la comparaison des deux cartes permet de quantifier les changements d'utilisation des terres liés aux pratiques agricoles adoptées par les populations locales (tableau II).

La première modification importante concerne la diminution des forêts et des savanes qui sont passées de 86,6 ha à 80,1 ha entre avril et juillet 2015. Les 6,5 ha de forêts et savanes manquants ont été défrichés (4,2 ha), voire déjà

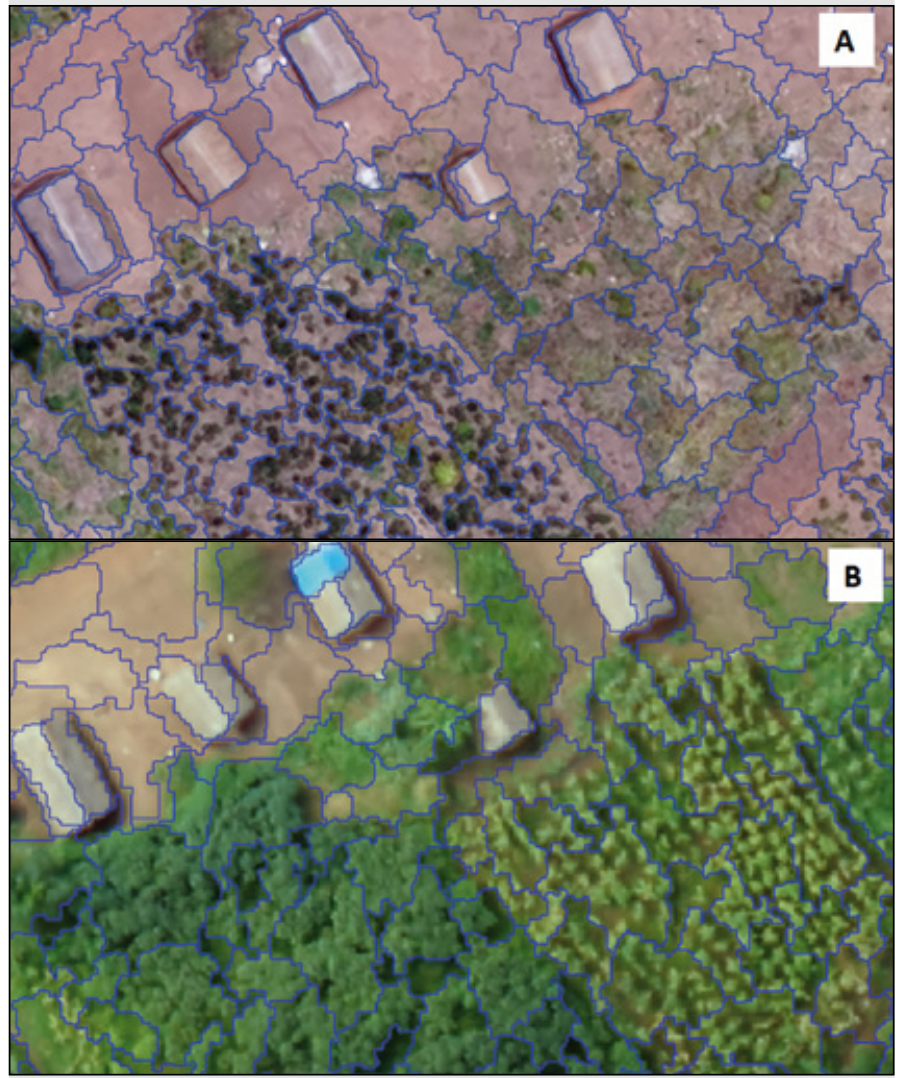

Figure 5.

Illustration du processus de segmentation des ortho-images à l'aide du logiciel eCognition Developer 9 : (a) pour l'image d'avril et (b) pour l'image de juillet.

mis en culture (1,4 ha), le solde (0,9 ha) étant constitué d'arbres ou de bouquets d'arbres isolés dans la mosaïque agricole. Une deuxième évolution importante porte sur les jachères qui sont passées de 16,9 ha à 8,2 ha, soit une diminution de plus de $50 \%$. Les surfaces concernées se répartissent de manière quasi uniforme entre cultures saisonnières (4,6 ha) et zones défrichées (4,8 ha). Si l'on s'intéresse à la nature des jachères transformées au cours de cette période, on constate que plus de $36 \%$ sont des jeunes jachères (tableau III).

La troisième évolution a trait aux cultures saisonnières. Leurs surfaces sont passées de 3,2 ha à 11,8 ha (tableau IV). En avril, elles sont exclusivement constituées de manioc alors qu'en juillet, toute la diversité des cultures saisonnières pratiquées par les villageois est représentée : manioc $(25,3 \%)$, riz (11,6\%), maïs (8,9\%), haricot $(6,3 \%)$, arachide $(0,7 \%)$, patate douce $(0,6 \%)$... Certaines des cultures sont exploitées en association, par exemple arachide-maïs, haricot-riz, manioc-arachide, ou encore riz-maïs. Cette évolution traduit le fait que la première date d'observation correspond à la fin de la campagne agricole de la saison précédente (fin 2014) et au début des travaux de la saison suivante (2015). La classe des arbres isolés reste relativement stable ; elle passe de 1,26 ha à 1,94 ha. Les surfaces occupées par les cultures pérennes peuvent également être considérées comme constantes ( 0,45 ha contre $0,46 \mathrm{ha})$. C'est également le cas des implantations humaines $(1,73$ ha en avril 
contre 1,75 ha en juillet). Cependant, pour ces dernières, treize des 107 maisons cartographiées en avril ont disparu en juillet, alors que quatorze nouvelles implantations sont détectées sur l'ortho-image de juillet, conduisant à un effectif total à peu près constant.

Notons enfin que la taille moyenne des parcelles cultivées à Gbere est de 0,20 ha ( $\sigma=0,14$ ha ; $\mathrm{n}=50)$.

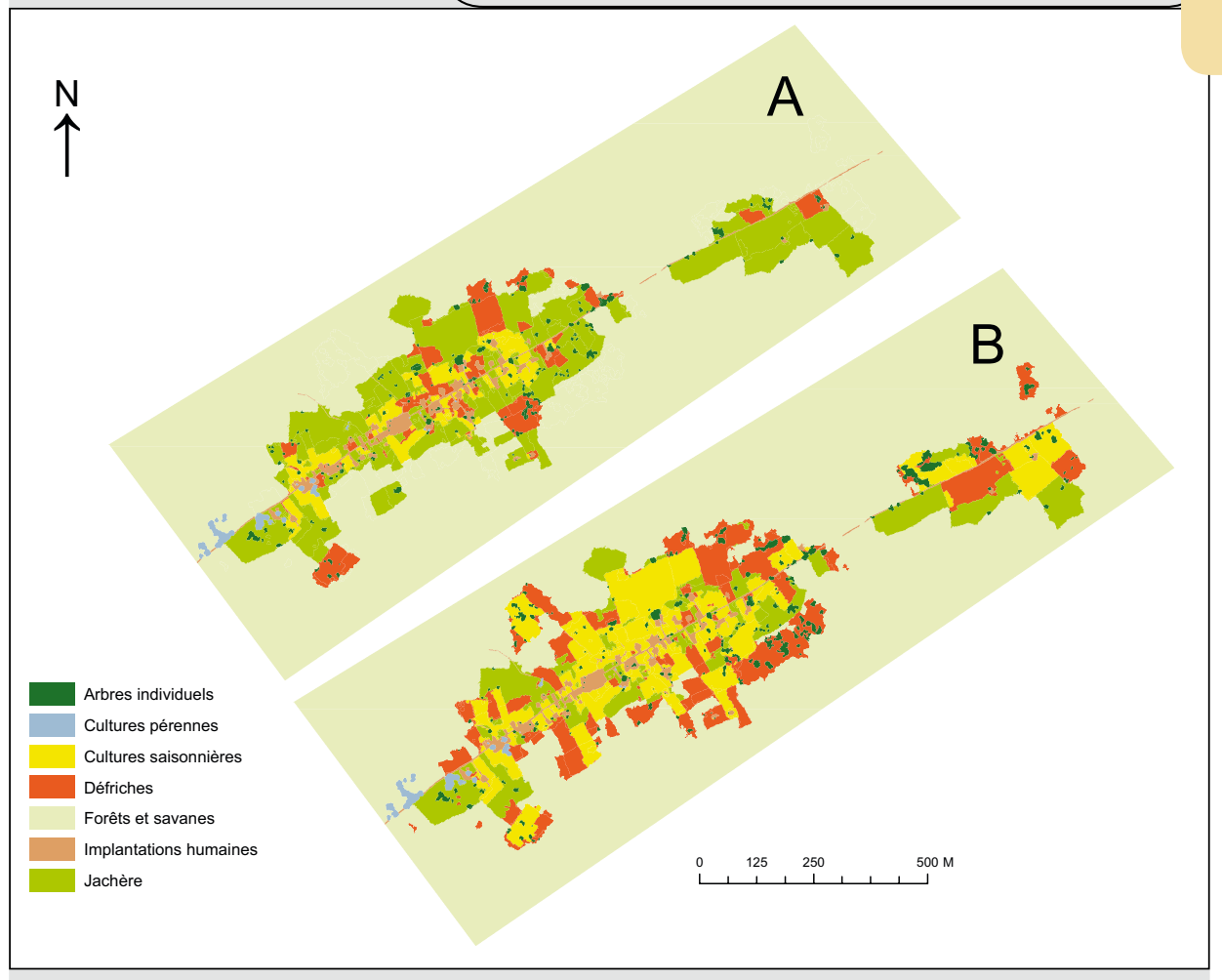

Figure 6.

Dynamique du terroir villageois de Gbere en 2015 entre (A) avril et (B) juillet.

\section{Tableau II.}

Matrice de transition de l'occupation des sols (surfaces exprimées en ha et en \%) entre avril 2015 et juillet 2015 pour le village de Gbere.

\begin{tabular}{|c|c|c|c|c|c|c|c|c|}
\hline \multirow[t]{2}{*}{ Surface (ha) } & \multirow[b]{2}{*}{ Arbres isolés } & \multirow[b]{2}{*}{$\begin{array}{l}\text { Cultures } \\
\text { pérennes }\end{array}$} & \multirow[b]{2}{*}{$\begin{array}{c}\text { Cultures } \\
\text { saisonnières }\end{array}$} & \multicolumn{2}{|c|}{ Juillet 2015} & \multirow[b]{2}{*}{$\begin{array}{l}\text { Implantations } \\
\text { humaines }\end{array}$} & \multirow[b]{2}{*}{ Jachères } & \multirow[b]{2}{*}{ Total } \\
\hline & & & & Défriches & $\begin{array}{l}\text { Forêts et } \\
\text { savanes }\end{array}$ & & & \\
\hline \multicolumn{9}{|l|}{ Avril 2015} \\
\hline Arbres isolés & $\begin{array}{c}1,02 \\
(80,9)\end{array}$ & & $\begin{array}{c}0,15 \\
(11,9)\end{array}$ & $\begin{array}{l}0,08 \\
(6,4)\end{array}$ & & $\begin{array}{l}0,01 \\
(0,8)\end{array}$ & & $\begin{array}{c}1,26 \\
(100,0)\end{array}$ \\
\hline $\begin{array}{l}\text { Cultures } \\
\text { pérennes }\end{array}$ & & $\begin{array}{c}0,45 \\
(100,0)\end{array}$ & & & & & & $\begin{array}{c}0,45 \\
(100,0)\end{array}$ \\
\hline $\begin{array}{l}\text { Cultures } \\
\text { saisonnières }\end{array}$ & & & $\begin{array}{c}2,76 \\
(85,7)\end{array}$ & $\begin{array}{l}0,10 \\
(3,1)\end{array}$ & & & $\begin{array}{c}0,36 \\
(11,2)\end{array}$ & $\begin{array}{c}3,21 \\
(100,0)\end{array}$ \\
\hline Défriches & & & $\begin{array}{c}2,89 \\
(71,2)\end{array}$ & $\begin{array}{c}0,83 \\
(20,4)\end{array}$ & & & $\begin{array}{l}0,34 \\
(8,4)\end{array}$ & $\begin{array}{c}4,06 \\
(100,0)\end{array}$ \\
\hline $\begin{array}{l}\text { Forêts et } \\
\text { savanes }\end{array}$ & $\begin{array}{l}0,92 \\
(1,1)\end{array}$ & & $\begin{array}{l}1,35 \\
(1,6)\end{array}$ & $\begin{array}{l}4,20 \\
(4,8)\end{array}$ & $\begin{array}{l}80,10 \\
(92,5)\end{array}$ & & & $\begin{array}{c}86,57 \\
(100,0)\end{array}$ \\
\hline $\begin{array}{l}\text { Implantations } \\
\text { humaines }\end{array}$ & & & $\begin{array}{l}0,01 \\
(0,6)\end{array}$ & & & $\begin{array}{c}1,72 \\
(99,4)\end{array}$ & & $\begin{array}{c}1,73 \\
(100,0)\end{array}$ \\
\hline Jachères & & $\begin{array}{l}0,01 \\
(0,1)\end{array}$ & $\begin{array}{c}4,64 \\
(27,4)\end{array}$ & $\begin{array}{c}4,79 \\
(28,3)\end{array}$ & & $\begin{array}{l}0,01 \\
(0,1)\end{array}$ & $\begin{array}{c}7,47 \\
(44,1)\end{array}$ & $\begin{array}{c}16,92 \\
(100,0)\end{array}$ \\
\hline Total & $\begin{array}{l}1,94 \\
(1,7)\end{array}$ & $\begin{array}{l}0,46 \\
(0,4)\end{array}$ & $\begin{array}{l}11,81 \\
(10,3)\end{array}$ & $\begin{array}{l}9,98 \\
(8,8)\end{array}$ & $\begin{array}{l}80,10 \\
(70,1)\end{array}$ & $\begin{array}{l}1,75 \\
(1,5)\end{array}$ & $\begin{array}{l}8,16 \\
(7,2)\end{array}$ & $\begin{array}{l}114,20 \\
(100,0)\end{array}$ \\
\hline
\end{tabular}




\section{Discussion}

\section{Efficacité des opérations}

L'approche cartographique développée à partir d'images collectées par drones pour décrire la dynamique du terroir villageois sur le site d'étude semble efficace. Les images ont été acquises en une seule journée par période, ce qui peut être considéré comme acceptable en termes de délai d'exécution. Les limitations concernant l'endurance des mini-drones à propulsion électrique (Rango et al., 2009 ; Linchant et al., 2015) ne se sont donc pas avérées être un problème dans le cas présent.
Les résultats du géoréférencement et de l'assemblage des images démontrent que l'altitude de vol et les recouvrements d'image retenus permettent d'éviter les difficultés parfois rencontrées dans la phase d'assemblage des images acquises, particulièrement en zone forestière (Koh et Wich, 2012 ; Semeki et al., 2016). La démarche d'ortho-rectification et de création des ortho-images développée ne nécessite pas beaucoup de temps de travail pour l'opérateur. La qualité des ortho-photos produites est suffisante pour documenter de manière précise les changements de l'occupation des sols à l'échelle du terroir agricole. En effet, Wu Jun et al. (2007) ainsi que Laliberte et al. (2010) ont montré que des erreurs de l'assemblage de 1-2 pixels étaient acceptables si l'on considère l'instabilité des mini-drones et la distorsion des caméras numériques utilisées.

\section{Tableau III.}

Nature des jachères transformées entre avril 2015 et juillet 2015 (surfaces exprimées en ha et en \%).

\begin{tabular}{|c|c|c|c|}
\hline Surfaces (ha) & & Situation en juillet 201 & \\
\hline Type de jachère en avril 2015 & Défriches & Cultures saisonnières & Total \\
\hline Jeunes jachères & 1,55 & 1,93 & $\begin{array}{c}3,48 \\
(36,1 \%)\end{array}$ \\
\hline $\begin{array}{l}\text { Formations herbeuses avec } \\
\text { présence de Chromolaena odorata }\end{array}$ & 2,61 & 2,46 & $\begin{array}{c}5,07 \\
(53,8 \%)\end{array}$ \\
\hline $\begin{array}{l}\text { Formations herbeuses avec } \\
\text { présence d'un tapis graminéen }\end{array}$ & 0,42 & 0,22 & $\begin{array}{c}0,64 \\
(6,8 \%)\end{array}$ \\
\hline Vieille jachère & 0,20 & 0,02 & $\begin{array}{c}0,22 \\
(2,3 \%)\end{array}$ \\
\hline Total & 4,79 & 4,64 & $\begin{array}{c}9,43 \\
(100,0 \%\end{array}$ \\
\hline
\end{tabular}

Tableau IV.

Évolution des cultures saisonnières entre avril et juillet 2015 : surfaces cultivées (en ha et en \%).

\begin{tabular}{|c|c|c|c|c|}
\hline \multirow[t]{2}{*}{ Type de cultures saisonnières } & \multicolumn{2}{|c|}{ Avril 2015} & \multicolumn{2}{|c|}{ Juillet 2015} \\
\hline & ha & $\%$ & ha & $\%$ \\
\hline Arachide-maïs & & & 0,28 & 2,4 \\
\hline Arachide & & & 0,09 & 0,7 \\
\hline Haricot & & & 0,74 & 6,3 \\
\hline Haricot-riz & & & 0,35 & 3,0 \\
\hline Maïs & & & 1,05 & 8,9 \\
\hline Manioc & 3,10 & 96,5 & 2,99 & 25,3 \\
\hline Manioc-arachide & & & 0,06 & 0,3 \\
\hline Manioc-maïs & & & 0,50 & 4,2 \\
\hline Patate douce & & & 0,08 & 0,7 \\
\hline Patate douce-manioc-maïs & & & 0,01 & 0,1 \\
\hline Riz & & & 1,37 & 11,6 \\
\hline Riz-maïs & & & 0,60 & 5,1 \\
\hline Semis & 0,11 & 3,5 & 3,70 & 31,4 \\
\hline Total & 3,21 & 100,0 & 11,82 & 100,0 \\
\hline
\end{tabular}



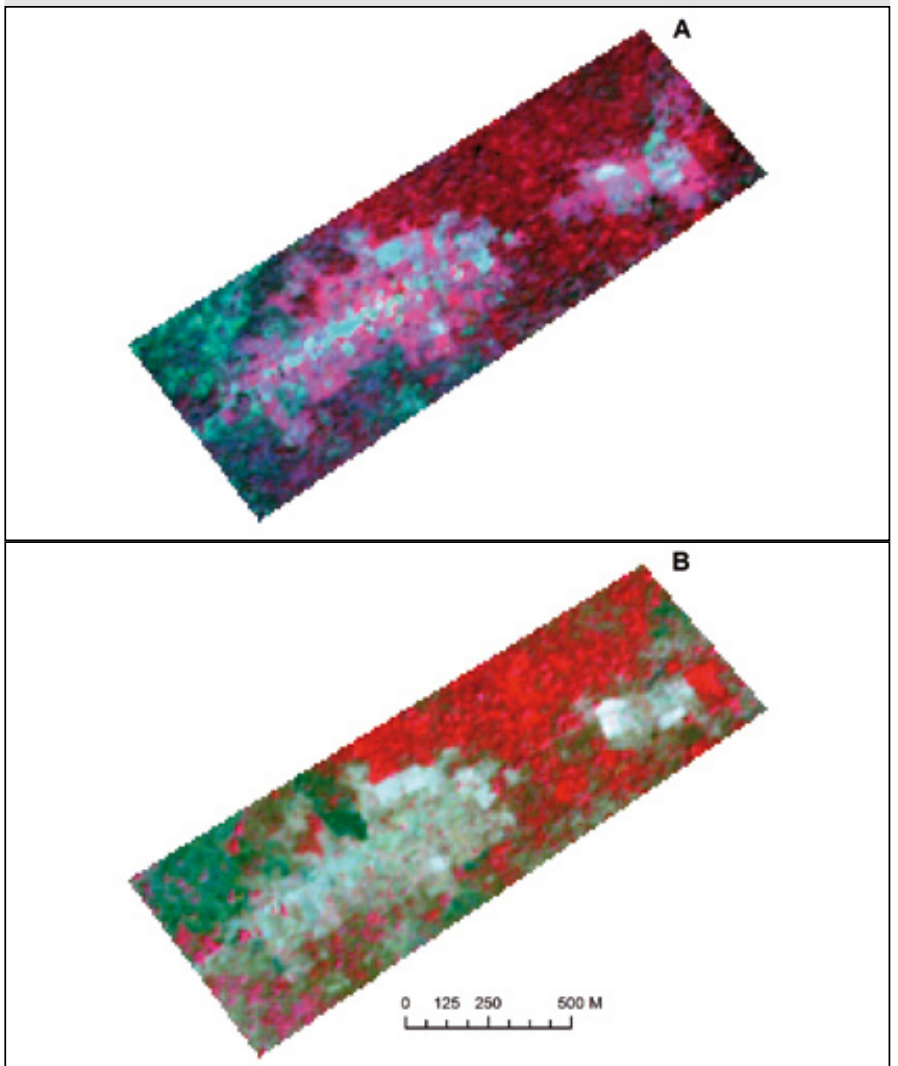

Figure 7.

Extrait d'images Sentinel-2 sur le village de Gbere (a) le 5 décembre 2015 et (b) le 13 février 2016. Les images sont affichées en composition colorée infrarouge fausses couleurs (RGB $=843)$. Source des données : https://scihub. copernicus.eu
Les paramètres de la segmentation utilisés ont permis de regrouper les pixels adjacents des différents objets homogènes, ce qui a facilité l'interprétation des images et le codage des polygones. L'importance du temps de travail consacré à ces deux tâches s'explique essentiellement par la grande hétérogénéité de l'occupation des sols et la complexité de la mosaïque de cultures saisonnières, de jachères, de défriches et d'habitations imbriquées dans la matrice forêts-savanes (Mayaux et al., 1999 ; Geist et Lambin, 2002 ; Molinario et al., 2015).

\section{Drone versus satellite haute résolution}

L'avènement de la nouvelle génération d'images Sentinel-2 produites par l'Agence spatiale européenne devrait probablement ouvrir de nouvelles possibilités en matière de cartographie et de monitoring des activités humaines au sein d'aires protégées (Nagendra et al., 2015). Elles sont accessibles gratuitement et sont caractérisées par une résolution spatiale de $10 \mathrm{~m}$ pour les bandes couramment utilisées en cartographie de la végétation (vert, rouge et proche infrarouge) et une résolution temporelle qui, à terme, devrait être de cinq jours (ESA, 2015).

À titre d'illustration, nous présentons des extraits d'images Sentinel-2 sur la zone d'étude acquises à deux dates, le 5 décembre 2015 et le 13 février 2016 (figure 7). L'intérêt de disposer de séries temporelles permettant d'appréhender les moments clés du calendrier agricole apparaît clairement en observant ces clichés. Même si ce type d'image présente un intérêt évident pour le suivi d'activités humaines au sein d'aires protégées, leur mise en œuvre soulève notamment un problème d'accessibilité dans un pays comme la RDC, les fichiers, d'une taille nominale de 5 à 6 Go, étant accessibles uniquement par Internet. À titre d'exemple, l'accès aux images représentées dans la figure 7 nécessite de télécharger environ 12 Go de données. En outre, la résolution spatiale de $10 \mathrm{~m}$ est loin d'offrir le degré de détail des images collectées avec des drones et d'égaler leur capacité à décrire de manière fine un parcellaire très morcelé.

On peut considérer que les images satellitaires de type Sentinel-2 offrent un réel intérêt lorsque l'on souhaite couvrir de grandes surfaces (au-delà de $10^{4} \mathrm{ha}$ ), avec cependant des exigences limitées quant au niveau de détail, et moyennant l'existence d'une infrastructure de communication performante.

Pour sa part, la filière d'acquisition de données acquises avec des drones ne peut couvrir que des surfaces limitées (de $10^{2}$ ha à $10^{4}$ ha). En revanche, elle produit des images avec un niveau de détail difficilement égalable et une fréquence d'acquisition qui peut être élevée, sans nécessiter aucune infrastructure de télécommunication. II s'agit d'un mode de production et d'analyse de données en circuit court, particulièrement adapté à des pays comme la RDC.
Figure 8.

Détection des changements de l'occupation des sols du terroir de Gbere entre avril et juillet 2015, caractérisés par la conversion et la fragmentation de l'habitat de l'aire protégée. 


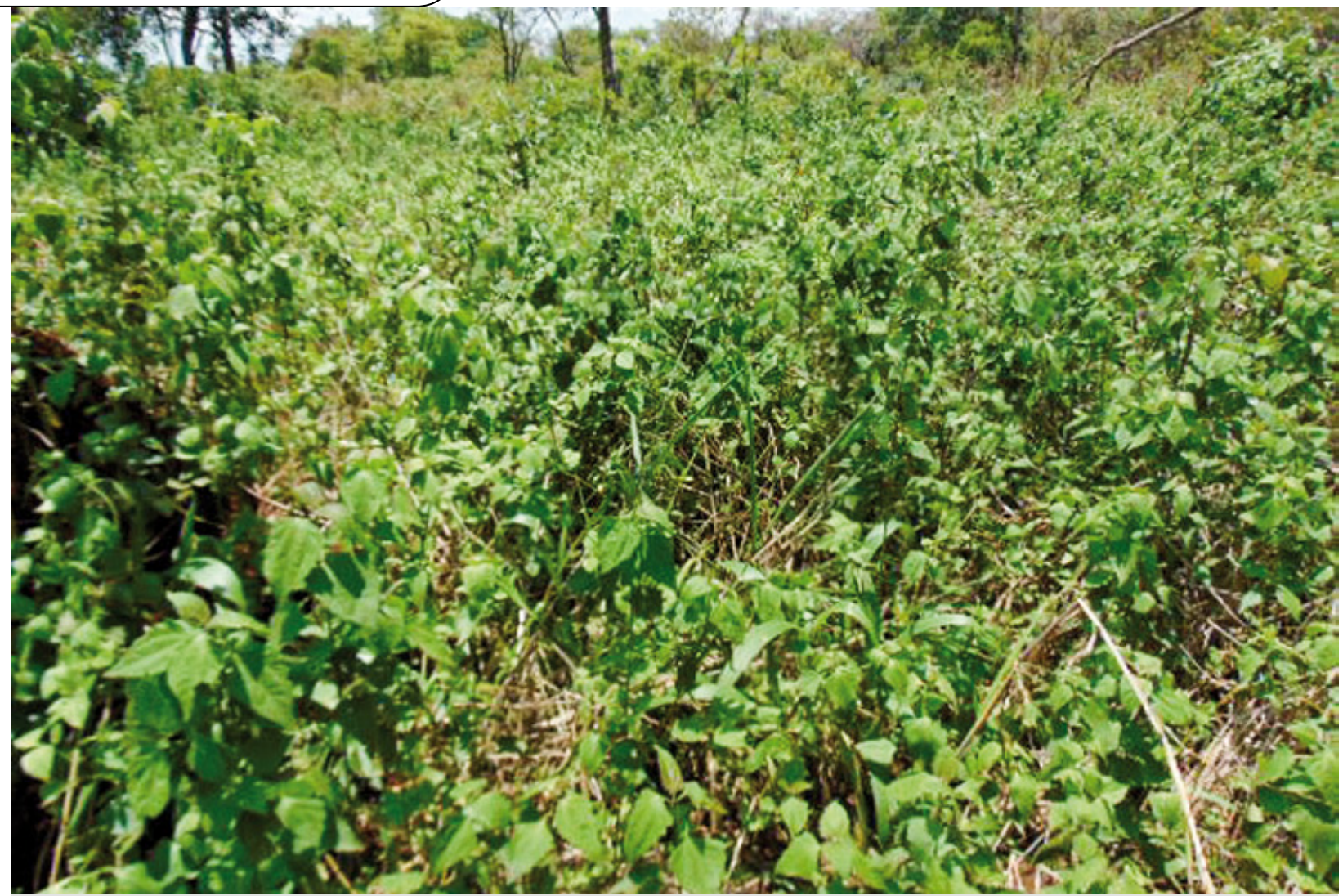

Photo 2.

Jachère herbeuse avec présence de Chromolaena odorata à Gbere.

Photo J. Semeki Ngabinzeke.

La caméra RVB utilisée dans cette étude fournit des images en couleurs vraies (spectre lumineux du visible pour l'œil humain) peu propices à l'utilisation d'algorithmes de classification automatique. L'apparition de capteurs multispectraux compacts, utilisables sur des mini-drones, ouvre des perspectives intéressantes pour l'application des outils de télédétection classiques (calcul d'indices de végétation, classification supervisée) aux images acquises par drone (Berni et al., 2009 ; Turner et al., 2011 ; Baluja et al., 2012). Cependant, ces capteurs sont généralement plus encombrants, plus onéreux, avec une résolution spatiale moindre que les caméras RVB classiquement employées sur les minidrones (Paneque-Gálvez et al., 2014 ; Whitehead et Hugenholtz, 2014).

Pour être complet dans cette comparaison, il convient de mentionner que la mise en œuvre des drones est également confrontée au respect d'exigences réglementaires (Hardin et Jensen, 2011 ; Watts et al., 2012). Dans un pays comme la RDC, déchiré par des conflits armés depuis de nombreuses années, les drones conservent une très forte connotation militaire et leur déploiement sur la zone d'étude a nécessité de nombreuses autorisations de la part de plusieurs autorités administratives, car par exemple les fréquences radio utilisées par les drones peuvent être réservées pour d'autres applications. Une vigilance est à observer avant la mise en œuvre car dans certains pays, l'appareil peut être considéré, suivant la réglementation en vigueur, comme un appareil d'espionnage.

\section{types de pression induite sur l'habitat et variabilité des cultures}

Les résultats obtenus sur l'analyse de la dynamique du terroir villageois de Gbere démontrent que les ortho-images produites constituent des cartes thématiques à très haute résolution spatiale. Ces données permettent de détecter et de surveiller rapidement les changements d'état de l'occupation des sols à l'échelle locale et d'évaluer leur évolution en termes de surfaces perturbées. En effet, Nagendra et al. (2015) ont établi quatre grandes catégories de changements d'état permettant d'identifier les types de pression anthropique dans une aire protégée. Parmi ces changements, la pratique de l'agriculture itinérante sur brûlis dans la zone d'étude est à la base de la conversion et de la fragmentation de l'habitat (figure 8) mais aussi de la modification de la structure de la communauté végétale. La conversion de l'habitat est observée par la perte du couvert forestier et savanicole présent en avril et sa transformation en défriches et cultures saisonnières en juillet. Ces perturbations sont également à l'origine de la fragmentation du couvert forestier, caractérisée par l'existence de reliques d'arbres isolés ou de bosquets. Le corollaire de ce fractionnement est la diminution de la connectivité du couvert arboré dans le paysage et la dégradation de l'habitat naturel (Nackoney et al., 2014 ; Ickowitz et al., 2015).

La présence de Chromolaena odorata (photo 2) comme l'une des plantes prédominantes de jachères herbeuses est un signe de la transformation de la composition floristique 
initiale. Cette espèce exotique envahissante ne fait pas partie de la végétation indigène du complexe de la Garamba (De Saeger, 1954). Elle constitue une menace directe pour la conservation car elle réduit la diversité de la végétation de forêts et savanes en freinant le développement des espèces locales (Mandisa et al., 2008).

Les résultats obtenus montrent que les drones constituent une solution pertinente pour cartographier de manière précise la dynamique d'occupation des sols à l'échelle des terroirs villageois. Ils pourraient donc représenter un outil de monitoring intéressant pour le suivi de la mise en œuvre des paiements pour services environnementaux (PSE) dans le cadre du processus REDD+ (Réduction des émissions dues à la déforestation et la dégradation des forêts) à l'échelle des communautés locales (Paneque-Gálvez et al., 2014 ; Kakaes et al., 2015 ; Karsenty, 2015) dans la mesure où ils permettent d'appréhender des modifications d'occupation des sols à l'échelle des besoins de l'unité familiale.

En outre, cette étude a mis en évidence une dynamique saisonnière marquée par une réduction de la durée des jachères et une augmentation de la diversité culturale entre avril et juillet 2015. Ces faits corroborent les conclusions d'Angelsen et Kaimowitz (2001) attestant que le cycle de l'agriculture itinérante sur brûlis se caractérise parfois par l'expansion des cultures en zone forestière et par la surexploitation des jachères. Molinario et al. (2015) ont démontré qu'en RDC le cycle de la culture itinérante varie en fonction des différents facteurs (environnementaux, socio-économiques, culturels, politiques) observés dans une zone donnée. Le système cultural «Gbere » est d'abord influencé par le climat écologique local, ce qui explique les différences d'occupation des sols observées aux deux dates. La grande saison sèche (décembre-février) est caractérisée par une très forte insolation, des variations thermiques, une évaporation et une dessiccation par les vents secs, tous ces éléments altérant le bilan en eau des cultures (De Saeger, 1954). Lors des entretiens, les populations ont évoqué l'absence de précipitations en mars 2015 et la prolongation de la saison sèche jusqu'au début d'avril, ce qui ne leur a pas permis d'emblaver. D'autre part, les attaques récurrentes du groupe armé de la LRA (Armée de Résistance du Seigneur) dans la zone d'étude amènent les populations à fuir leur village et à s'installer aux endroits relativement sécurisés (Unesco, 2010 ; Balimbaki, 2015). Pour s'adapter à cette situation et subvenir à leurs besoins quotidiens, les populations déplacées sont obligées de pratiquer les cultures de courte durée (haricot, maïs, riz, arachide) pour leur permettre d'écouler rapidement les produits. Gbere est situé à environ $20 \mathrm{~km}$ du centre urbain de Farage où vivent des milliers de déplacés qui viennent cultiver sur le site de Gbere. L'évolution du nombre de maisons confirme également les mouvements de populations et constitue un autre indicateur des effets de la guerre dans cette partie du pays. Nackoney et al. (2014) et Butsic et al. (2015) ont rapporté qu'à l'est et au nord de la RDC les déplacés, pour éviter les milices, vont s'installer au cœur des aires protégées qui constituent un lieu de refuge et de survie. Ces mutations ont ainsi aggravé les pressions sur celles-ci et ont conduit à la fragmentation des forêts intactes (Molinario et al., 2015).
L'intensité des changements d'occupation des sols, observée dans un laps de temps court (6 mois), est donc pour partie liée au cycle cultural local, mais probablement aussi, pour une autre partie, à une tendance à l'augmentation des mouvements de populations au sein du complexe de la Garamba. Un suivi régulier et pluriannuel de cette zone permettrait de mieux cerner cet effet plus conjoncturel susceptible d'entraîner une augmentation importante de la dégradation du milieu au sein de cette aire protégée.

Enfin, la taille moyenne des parcelles cultivées à Gbere avoisine celle trouvée par Wilkie et Finn (1990) dans la forêt d'Ituri $(0,25 \mathrm{ha})$, alors que la moyenne nationale s'élève à 1,4 ha (Potapov et al., 2012). La faible étendue des zones défrichées confirme l'utilité des drones pour surveiller les pressions anthropiques dans un tel contexte (Nagendra et al., 2013 ; Kakaes et al., 2015).

\section{Conclusion}

Cette étude démontre que de petits drones équipés d'un appareil photo numérique à faible coût peuvent être utilisés avec succès pour cartographier et surveiller rapidement et fréquemment la dynamique des terroirs villageois dans les aires protégées de forêts et savanes tropicales. Les images à très hautes résolutions spatiale et temporelle qu'ils fournissent permettent d'opérer rapidement une analyse précise des changements de l'utilisation des terres et du type de pression induits sur les aires protégées. Dans ce cas, les performances de cet outil dépassent ce qui est envisageable avec les données de télédétection aéroportée ou satellitaire. Par contre, les limitations des drones en matière d'endurance ne permettent pas de rivaliser avec les satellites tels que Sentinel-2, dès lors que les surfaces à couvrir deviennent trop importantes (au-delà de $10^{4} \mathrm{ha}$ ). Les gestionnaires des parcs et les acteurs de la conservation disposent néanmoins d'un outil intéressant d'aide à la décision pour améliorer la gouvernance et les politiques de gestion territoriale à l'échelle de communautés locales. Par ailleurs, ces niveaux de performances permettent d'envisager l'usage des drones pour le suivi des défrichements dans le cadre des processus REDD+, dans la mesure où de faibles variations de surfaces agricoles peuvent être détectées à l'échelle de l'unité familiale. Enfin, s'agissant de perspectives, les images acquises devraient permettre ultérieurement des approches liées à l'écologie du paysage, comme le calcul de différents indices relatifs à la fragmentation et la connectivité des différents habitats constitutifs de ces écosystèmes (Faye et al., 2015).

\section{Remerciements}

Cette recherche fait partie intégrante du projet WiMUAS (Wildlife Monitoring with Unmanned Aerial Systems) mis en œuvre par Gembloux Agro-Bio Tech avec le financement du Centre pour la recherche forestière internationale (CIFOR) et de l'Union européenne dans le cadre du projet Forêts et changement climatique au Congo (FCCC), auxquels les auteurs tiennent à exprimer leur reconnaissance. Nos remerciements vont également au bureau d'études R\&SD (Resources \& Synergies Development) pour son appui 
logistique très précieux dans la réalisation de cette étude. Enfin, nous exprimons notre gratitude à l'ICCN et l'African Parks Network pour avoir accueilli ce projet au Parc national de la Garamba et pour leur soutien logistique et sécuritaire lors de la phase de collecte des données.

\section{Références bibliographiques}

Angelsen A., Kaimowitz D., 2001. When does technological change in agriculture promote deforestation? In: Lee D. R., Barrett C. B. (eds). Tradeoffs or Synergies ? Agricultural Intensification, Economic Development and the Environment. Wallingford, United Kingdom, CABI, 89-114.

Balimbaki L. A., 2015. Étude socio-économique dans les trois domaines de chasse contigus au Parc National de la Garamba. Nagero, République démocratique du Congo, ICCN-APN, Département recherche et monitoring, $76 \mathrm{p}$.

Baluja J., Diago M. P., Balda P., 2012. Assessment of vineyard water status variability by thermal and multispectral imagery using an unmanned aerial vehicle (UAV). Irrigation Science, 30: 511-522.

Berni J. A. J., Zarco-Tejada P. J., Suárez L., Fereres E., 2009. Thermal and narrowband multispectral remote sensing for vegetation monitoring from an unmanned aerial vehicle. IEEE Transactions on Geoscience and Remote Sensing, 473: 722-738.

Butsic V., Baumann M., Shortland A., Walker S., Kuemmerle T., 2015. Conservation and conflict in the Democratic Republic of Congo: the impacts of warfare, mining, and protected areas on deforestation. Biological Conservation, 191: 266-273.

De Saeger Z. H., 1954. Exploration du Parc National de la Garamba : Introduction. Mission de Z. H. De Saeger, en collaboration avec P. Baert, G. Demoulin, I. Denisoff, J. Martin, M. Micha, A. Noirfalise, P. Schoemarker, G. Troupin et J. Verschuren. Institut des Parcs Nationaux du Congo Belge. Bruxelles, Belgique, Imprimerie Hayez, 107 p.

Eba’a Atyi R., Bayol N., 2009. Les forêts de la République Démocratique du Congo en 2008. In : de Wasseige C., Devers D., de Marcken P., Eba'a Atyi R., Nasi R., Mayaux P. (éds). Les forêts du Bassin du Congo - État des forêts 2008. Luxembourg, Office des publications de l'Union européenne, 115-128.

ESA, 2015. Sentinel-2_User_Handbook. ESA. https://sentinel.esa.int/documents/247904/685211/Sentinel-2 User Handbook (consulté le 13 mai 2016).

Faye E., Rebaudo F., Yánez-Cajo D., Cauvy-Fraunié S., Dangles O., 2015. A toolbox for studying thermal heterogeneity across spatial scales: from unmanned aerial vehicle imagery to landscape metrics. Methods in Ecology and Evolution, 7 (4): 437-446.

Geist H. J., Lambin E. F., 2002. Proximate causes and underlying driving forces of tropical deforestion. BioScience, 52 (2): 143-150.

Getzin S., Wiegand K., Schöning I., 2012. Assessing biodiversity in forests using very high-resolution images and unmanned aerial vehicles. Methods in Ecology and Evolution, 3 (2): 397-404.
Hardin P. J., Jensen R. R., 2011. Small-scale unmanned aerial vehicles in environmental remote sensing: challenges and opportunities. GIScience \& Remote Sensing, 48 (1): 99-111.

ICCN, 2012. Stratégie nationale de conservation de la biodiversité dans les aires protégées de la République Démocratique du Congo. Kinshasa, RDC, Institut congolais pour la conservation de la nature, $18 \mathrm{p}$.

Ickowitz A., Slayback D., Asanzi P., Nasi R., 2015. Agriculture and deforestation in the Democratic Republic of the Congo: A synthesis of the current state of knowledge. Bogor, Indonesia, CIFOR, Occasional Paper 119, 18 p.

Kakaes K., Greenwood F., Lippincott M., Meier P., Wich S., 2015. Drones and aerial observation: New technologies for property rights, human rights, and global development. A primer. Washington, USA, New America, 104 p.

Karsenty A., 2015. Mettre les PSE au service de l'agriculture « zéro déforestation ». Montpellier, France, Cirad, Perspective n 36.

Kayungura G. T., 2009. Enquêtes socio-économiques et d'attitudes des populations riveraines autour du Parc National de la Garamba. Nagero, République démocratique du Congo, Fauna and Flora International and African Parks Network, 69 p.

Koh L. P., Wich S. A., 2012. Dawn of drone ecology: low-cost autonomous aerial vehicles for conservation. Tropical Conservation Science, 5 (2): 121-132.

Laliberte A. S., Jeffrey E. H., Rango A., Winters C., 2010. Acquisition, orthorectification and object-based classification of unmanned aerial vehicle (UAV) imagery for rangeland monitoring. Photogrammetric Engineering \& Remote Sensing, 76 (6): 661-672.

Larzillière A., Vermeulen C., Dubiez E., Yamba Yamba T., Diowo S., Mumbere G., 2013. La maquette interactive, un outil novateur de participation. Bois et Forêts des Tropiques, 315 (1) : 21-28. http://bft.cirad.fr/cd/BFT 315 21-28.pdf

Linchant J., Lisein J., Semeki J., Lejeune P., Vermeulen C., 2015. Are unmanned aircraft systems (UAS) the future of wildlife monitoring? A review of the accomplishments and challenges. Mammal Review, 45: 239-252.

Mandisa P. M., Somers M. J., Dippenaar-Schoeman A. S., 2008. Spider responses to alien plant invasion: the effect of short-and long-term Chromolaena odorata invasion and management. Journal of Applied Ecology, 45: 1189-1197.

Mayaux P., Richards T., Janodet E., 1999. A vegetation map of Central Africa derived from satellite imagery. Journal of Biogeography, 26: 353-366.

Merino L., Caballero F., Martinez-de-Dios J. R., Maza I., Ollero A., 2012. An unmanned aircraft system for automatic forest fire monitoring and measurement. Journal of Intelligent \& Robotic Systems, 65 (1): 533-548.

Misser F., 2013. Les aires protégées en République Démocratique du Congo : menaces et défis. L'action de l'Union européenne. Parcs \& Réserves, 68 (3) : 1-51.

Molinario G., Hansen M. C., Potapov P. V., 2015. Forest cover dynamics of shifting cultivation in the Democratic Republic of Congo: a remote sensing-based assessment for 2000-2010. Environmental Research Letters, 10 (9). 
Nackoney J., Molinario G., Potapov P., Turubanova S., Hansen M. C., Furuichi T., 2014. Impacts of civil conflict on primary forest habitat in northern Democratic Republic of the Congo, 19902010. Biological Conservation, 170: 321-328.

Nagendra H., Lucas R., Honrado J. P., Jongman R. H. G., Tarantino C., Adamo M., Mairota P., 2013. Remote sensing for conservation monitoring: assessing protected areas, habitat extent, habitat condition, species diversity, and threats. Ecological Indicators, 33: 45-59.

Nagendra H., Mairota P., Marangi C., Lucas R., Dimopouluos P., Honrado J. P. et al., 2015. Satellite Earth observation data to identify anthropogenic pressures in selected protected areas. International Journal of Applied Earth Observation and Geoinformation, 37: 124-132.

Paneque-Gálvez J., McCall M. K., Napoletano B. M., Wich S. A., Koh L. P., 2014. Small drones for Community-Based Forest Monitoring: An assessment of their feasibity and potential in tropical areas. Forests, 5: 1481-1507.

Pélissier C., De Marcken P., Mapilanga wa Tsaramu J. J., Wilungula B. C., 2015. République Démocratique du Congo. In : Doumenge C., Palla F., Scholte P., Hiol Hiol F., Larzillière A. (eds). Aires protégées d'Afrique centrale - État 2015. Kinshasa, République démocratique du Congo, Yaoundé, Cameroun, OFAC, 111-147.

Phalan B., Bertzky M., Butchart S. H. M., Donald P. F., Scharlemann J. P. W., Stattersfield A. J., Balmford A., 2013. Crop expansion and conservation priorities in tropical countries. PloS ONE 8 (1): e51759.

Potapov P. V., Turubanova S. V., Hansen M. C., Adusei B., Broich M., Altstatt A., Mane L., Justice C. O., 2012. Quantifying forest cover loss in Democratic Republic of the Congo, 2000-2010, with Landsat ETM+ data. Remote Sensing of Environment, 122: 106-116.

Puttock A. K., Cunliffe A. M., Anderson K., Brazier R. E., 2015. Aerial photography collected with a multirotor drone reveals impact of Eurosian beaver reintroduction on ecosystem structure. Journal of Unmanned Vehicle Systems, 3 (3): 123-130.

Rango A., Laliberte A., Herrick J. E., Winters C., Havstad K., Steele C., Browning D., 2009. Unmanned aerial vehicle-based remote sensing for rangeland assessment monitoring and management. Journal of Applied Remote Sensing, 3: 033542.

Semeki N. J., Linchant J., Quevauvillers S., Kahindo M. J.-P., Lejeune P., Vermeulen C., 2016. Potentiel des véhicules aériens sans pilote dans la détection des activités humaines illégales dans les aires protégées en République Démocratique du Congo. Journal of Unmanned Vehicle Systems, 4 (2) : 151-159.

Shahbazi M., Théau J., Ménard P., 2014. Recent applications of unmanned aerial imagery in natural resource management. GIScience \& Remote Sensing, 51: 339-365.

Spanhove T., Vanden Borre J., Delalieux S., Haest B., Paelinckx D., 2012. Can remote sensing estimate fine-scale quality indicators of natural habitats? Ecological Indicators, 18: 403-412.

Svancara L., Scott J. M., Loveland T. R., Pidgorna A. B., 2009. Assessing the landscape context and conversion risk of protected areas using satellite data products. Remote Sensing of Environment, 113: 1357-1369.
Torres-Sánchez J., López-Granados F., Serrano N., Arquero O., Peña J. M., 2015. High-troughput 3-D monitoring of agricultural-tree plantations with unmanned aerial vehicle (UAV) technology. PloS ONE, 10 (6): e013079.

Turner D., Lucieer A., Watson C., 2011. Development of an unmanned aerial vehicle (UAV) for hyper resolution vineyard mapping based on visible, multispectral, and thermal imagery. Proceedings of the 34th International Symposium on Remote Sensing of Environment, Sydney, Australia, 10-15 April 2011.

UICN/PACO, 2010. Parcs et réserves de la République démocratique du Congo : évaluation de l'efficacité de gestion des aires protégées. Ouagadougou, Burkina Faso, UICN/PACO, 149 p.

Unesco, 2010. Patrimoine mondial dans le bassin du Congo. Paris, France, Centre du patrimoine mondial de l'Unesco, $64 \mathrm{p}$.

Wang Y., Mitchell B. R., Nugranad-Marzilli J., Bonynge G., Zhou Y., Shriver G., 2009. Remote sensing of land-cover change and landscape context of the National Parks: a case study of the northeast temperate network. Remote Sensing of Environment, 113: 1453-1461.

Watts A. C., Ambrosia V. G., Hinkley E. A., 2012. Unmanned aircraft systems in remote sensing and scientific research: classification and considerations of use. Remote Sensing, 4 (6): 1671-1692.

Wellens J., Midekor A., Traore F., Tychon B., 2013. An easy and low-cost method for preprocessing and matching small-scale amateur aerial photography for assessing agricultural land use in Burkina Faso. International Journal of Applied Earth Observation and Geoinformation, 23: 273-278.

Whitehead K., Hugenholtz C. H., 2014. Remote sensing of the environment with small unmanned aircraft systems (UASs). Part 1: A review of progress and challenges. Journal of Unmanned Vehicle Systems, 2 (3): 69-85.

Wiens J., Sutter R., Anderson M., Blanchard J., Barnette A., Anguilar-Amuchastegui N., Avery C., Laine S., 2009. Selecting and conservation lands for biodiversity: the role of remote sensing. Remote Sensing of Environment, 113: 1370-1381.

Wilkie D. S., Finn J. T., 1990. Slash-burn cultivation and mammal abundance in the Ituri forest, Zaïre. Biotropica, 22: 90-99.

Willis K. S., 2015. Remote sensing change detection for ecological monitoring in United States protected areas. Biological Conservation, 182: 233-242.

Wulder M. A., Hall R. J., Coops N. C., Franklin S. E., 2004. High spatial resolution remotely sensed data for ecosystem characterization. BioScience, 54 (6): 511-521.

Wu Jun, Zhongkui Dong, Zhigang Liu, Guoqing Zhou, 2007. Geo-registration and mosaic of UAV video for quick-response to forest fire disaster. In: Proceedings SPIE, vol. 6788, MIPPR 2007: Pattern Recognition and Computer Vision, 678810, November 15, 2007, Wuhan, China. 